\title{
INSERTION AND REMOVAL OF SUTURELESS AMNIOTIC MEMBRANES
}

\author{
Nicholas Colatrella, OD, FAAO, Dipl AAO, ABO, ABCMO \\ Owner and Medical Director, PineCone Vision Center, Sartell MN \\ Corresponding Author: NColatrella@pineconevisioncenter.com
}

Submitted: November 7, 2018. Accepted: December 18, 2018. Published: May 9, 2019.

\begin{abstract}
Treatments for ocular surface disease have evolved significantly over the past several years. Sutureless amniotic membranes (AMs) are easy to insert in the office and give practitioners the ability to monitor healing without interfering with the penetration of ophthalmic medications. With their ability to reduce inflammation, prevent scarring, and enhance epithelial proliferation, AM should be considered as a viable treatment option for managing patients with ocular surface disease.
\end{abstract}

Keywords: ocular surface disease, amniotic membrane, sutureless

The amniotic membrane (AM) is composed of an avascular, hypocellular stroma and an overlying basement membrane with a single layer of epithelial cells. ${ }^{1}$ It is derived from the innermost layer of the fetal membranes and contains collagen types IV, V and VII, fibronectin, laminins, proteoglycans, and glycosaminoglycans. They also contain several growth factors such as epidermal growth factor, fibroblast growth factor, transforming growth factor-beta and platelet-derived growth factor. ${ }^{2}$

AMs also appears to be an immune privileged tissue, because it does not express human leukocyte antigen (HLA)-A, B and C antigens, but contains some immunoregulatory factors, including HLA-G and Fas ligand. ${ }^{3}$ Therefore, it can be applied without the concern or fear of rejection.

AM has important anti-inflammatory, antiangiogenic, anti-scarring and antimicrobial properties. These effects promote epithelial cell migration into damaged areas before inflammation worsens and prior to scar tissue formation. ${ }^{4}$

While AM has traditionally been transplanted using sutures or fibrin glue in an operating room, the in-office application of self-retaining or sutureless amniotic membranes has emerged as a less invasive alternative, with promising clinical outcomes for patients with ocular surface disease.

Currently, two main types of AM are commercially available for in-office use: cryopreserved (epithelialized) and dehydrated (de-epithelialized). Both types come in a variety of options, including tissue thicknesses and diameters, depending on clinical needs.

The cryopreservation technique of AM involves slow freezing at $-80^{\circ} \mathrm{C}$ using DMEM/glycerol preservation media to allow for slow-rate freezing without ice formation. ${ }^{5}$ The tissue must remain frozen prior to use. Dehydrated AM is preserved using a vacuum with low-temperature heat to retain its cellular components. Unlike cryopreserved tissue, dehydrated AM is kept at room temperature.

ProKera (BioTissue) is a cryopreserved form of AM that must remain frozen prior to use. The membrane is secured around a polycarbonate ring or an elastomeric band and is inserted into the eye similar to large diameter contact lenses. ${ }^{5}$ AmbioDisk (Katena Products) is a dehydrated AM that is applied directly to the ocular 
surface and kept in place with an overlying bandage soft contact lens. There are currently several other manufacturers of dehydrated tissue including BioDOptix (Integra Life Sciences), Aril (Seed Biotech), etc., and all require the use of a bandage soft contact lens.

Most patients will tolerate the AM, but only if expectations are set prior to insertion. Temporary blurring of vision is expected, and some patients may experience mild to moderate discomfort. It's important to make patients aware of these potential difficulties and address them prior to utilization. ${ }^{6}$

\section{APPLICATION OF SUTURELESS AMNIOTIC MEMBRANES}

If you are just getting started in AM therapy, creating a standard routine for each patient will help you get acclimated. It is also recommended that you develop a consent form to be reviewed and signed prior to insertion.

When applying any membrane in the office, preparation is the key to success. Make sure you have all the appropriate pieces in place prior to opening the package or putting on a speculum. For cryopreserved membranes that means having a sterile wash on hand, and a place to drain the medium. For dehydrated membranes, you will want to have everything prepared (jewelers, speculum, sponge spears, soft contact lens) and ready prior to putting on the speculum. Having a couple of spare bandage soft contact lenses is never a bad idea.

Once applied, it is also good practice to keep the patient in the office for 10-20 minutes afterward. This will allow time for the membrane to settle, and help identify those patients that may be a little more sensitive to the membrane. After insertion, it is also good practice to prescribe and cover all membranes with a topical antibiotic (QID) along with or without a topical steroid.

\section{CRYOPRESERVED (ProKera)}

For cryopreserved AMs, remove the package from the freezer and start to prepare it for insertion. The preparation alone should allow enough time for the device to come to room temperature prior to application.

Open the box and gently remove the foil cover from the plastic base. Once opened, the ring will be clipped into the base with an overlying piece of plastic (keep this in place for now). Carefully turn the holder upside down to drain the preservation medium from the package.

Fill the fluid reservoirs in the plastic packaging back up with sterile saline and then drain. Repeat this several times to help rinse the glycerin off the ring / membrane. Repeat as needed.

Prior to insertion add a few drops of topical anesthetic to the ocular surface. Then, remove the plastic clip overlying the membrane. Insert the superior ring edge under the upper eyelid while the patient is looking down, and then have the patient look up while you slip the remainder of the ring onto the eye; the membrane is supplied in the correct orientation such that the stromal side will be in contact with the ocular surface.

If the ring and membrane create too much discomfort, or it appears the ring will dislodge. It is often helpful to place a small piece of tape over the upper lid to prevent it from opening completely. Be careful not to tape the lid completely shut, to allow the installation of medication as directed, and to avoid creating a warm moist environment where bacteria could grow.

The ring must be removed once the membrane has dissolved or hazed over. This will usually occur in 3-5 days but is dependent upon the amount of inflammation that is present. Careful observation of the patient while the membrane is in place will help you decide when to remove the ring.

Prior to removal, instill a few drops of topical anesthetic to the ocular surface. If needed, specialized forceps can help with the removal process. Have the patient look up and then lift the lower edge of the ring and grab at the 6 o'clock position. Gently slide in a downward direction and instruct the patient to look down at the same time. Avoid pulling up and away from the cornea when removing to prevent abrading the cornea as its removed. See Video 1 for further instruction.

\section{DEHYDRATED (AmbioDisk, BioD, Aril)}

All dehydrated membranes should be applied using a lid speculum or holding the eyes open to insert the membrane and soft contact lens. Insertion of an AM using a speculum may be new to some practitioners. 
Please see the Video 2 for a simple, four-step process that allows for easy insertion.

When selecting a membrane size, the $10-12-\mathrm{mm}$ diameter membranes are generally the most practical. Open the box and carefully remove the disk from the inner package - the dehydrated tissue is very thin and lightweight. There also tends to be a lot of static in the packaging bag, which can make the membrane stick to the package. Therefore, it is recommended to only open the bag halfway, to prevent the disk from inadvertently flying out.

Prior to insertion, instill a few drops of topical anesthetic to the ocular surface. It is recommended to apply the AM disk directly onto the ocular surface for the maximal therapeutic effect (rather than placing it into the soft contact lens and then onto the eye). When applying the AmbioDisk orient the graft with the "IOP" watermark facing the patient so the patient would read "IOP" to ensure that the stromal side is in contact with the eye.

Smooth the graft onto the ocular surface. However, due to the concavity of the corneal surface, there will be wrinkles in the AM that cannot be smoothed out.

Place a bandage soft contact lens to cover the membrane and corneal surface. Be sure the contact lens covers the membrane 360 degrees to ensure it does not get blinked out by the patient (i.e., use a 14$\mathrm{mm}$ soft contact lens over a 12-mm AM).

Typically, the stromal surface of the AMs is placed against the ocular surface. If ever in doubt, the correct side can be identified using a cellulose sponge, which sticks to the stroma, but not the epithelial surface.

The bandage soft contact lens must be removed once the membrane has dissolved or hazed over. This will usually occur in 3-5 days but is dependent upon the amount of inflammation that is present. Careful observation of the patient while the membrane is in place will help you decide when to remove the bandage soft contact lens.

\section{CONCLUSION}

Treatments for ocular surface disease have evolved significantly over the past several years. Sutureless AMs are easy to insert in the office, and they give practitioners the ability to monitor healing without interfering with the penetration of ophthalmic medications. With their ability to reduce inflammation, prevent scarring, and enhance epithelial proliferation, AM should be considered as a viable treatment option for managing patients with ocular surface disease.

\section{REFERENCES}

1. Dua HS, Gomes JA, King AJ, et al. The amniotic membrane in ophthalmology. Surv Ophthalmol 2004;49:51-77.

2. Rennie K, Gruslin A, Hengstschlager M, et al. Applications of amniotic membrane and fluid in stem cell biology and regenerative medicine. Stem Cells Int 2012; 2012:721538.

3. Kubo M, Sonoda Y, Muramatsu R, Usui M. Immunogenicity of human amniotic membrane in experimental xenotransplantation. Invest Ophthalmol Vis Sci 2001; 42:1539-46.

4. Tseng SCG, Espana EM, Kawakita T, et al. How does amniotic membrane work? Ocul Surf 2004;2:177-87.

5. Pachigolla G, Prasher P, Di Pascuale MA, et al. Evaluation of the role of ProKera in the management of ocular surface and orbital disorders. Eye Contact Lens 2009;35:172-75.

6. Ijiri S1, Kobayashi A, Sugiyama K, et al. Evaluation of visual acuity and color vision in normal human eyes with a sutureless temporary amniotic membrane patch. Am J Ophthalmol 2007;144(6):938-42. 